\title{
Automatic Control of Colonoscope Movement for Modern Colonoscopy
}

\author{
Helga Silaghi ${ }^{1}$, Viorica Spoiala ${ }^{2}$, Alexandru Marius Silaghi ${ }^{3}$, Tiberia Ioana Ilias ${ }^{4}$, Cornelia Győrödi $^{5}$, Claudiu Costea $^{6}$, \\ Sanda Dale ${ }^{7}$, Ovidiu Cristian Fratila ${ }^{8}$ \\ Faculty of Electrical Engineering and Information Technology, University of Oradea, Oradea, Romania $a^{1,2,3,5,6,7}$ \\ Medicine and Pharmacy Faculty, University of Oradea, Oradea, Romania ${ }^{4,8}$
}

\begin{abstract}
The paper presents the mathematical realization of the trajectory that the colonoscope should have in the medical intervention, as well as the mathematical demonstration of the functions that make up the colonoscope. The goal of this work is finding a method for reducing the medical doctor's effort, by using intelligent control of colonoscope movement for improving the comfort of the patient subjected to a classical colonoscopy and reducing the risk of perforation of the colon. Finally, some experimental results are presented, validating the model and the control solutions adopted in the paper.
\end{abstract}

Keywords-Intelligent control; colonoscope movement; classical colonoscopy; mathematical model

\section{INTRODUCTION}

Colon cancer is rarely seen before the age of 45 . After this age the mortality increases in 5 years with a ratio of $50 \%$. Statistically, this type of cancer occupies second place on the rate of death in both women (after breast cancer) and men (after lung cancer) [1] [6].

Colonoscopy is considered the reference exam because it allows to visualize the total colon surface and performs, if biopsy is required, by taking samples of polyps. Two elements predispose to the development of colon cancer: polyps with a particular clinical form and inflammatory diseases (hemorrhagic colitis, Chron malady). In addition to the two elements mentioned above, there are certain factors that predispose to the appearance of polyps: eating, the environment, sedentary, genetic factors, etc.

The goal of this work is finding a method for reducing the medical doctor's effort, by using intelligent control of colonoscope movement for improving the comfort of the patient subjected to a classical colonoscopy and reducing the risk of perforation of the colon.

\section{ARCHITECTURE AND FUNCTIONS OF COLONOSCOPE}

The human colon is a muscular organ of $1250 \mathrm{~mm}$ in length, which contributes to three major functions of the human body: the absorption of non-digested foods, the digestion and concentration of faeces, their storage and evacuation [6]. Functionally, the colon can be divided into two parts separated by the transverse portion (right colon and left colon), as shown in Fig. 1.

The right colon (or cecum and ascending colon) plays a major role in the absorption of water and electrolytes, but also in the fermentation of undigested sugar. The left colon (the colon, the sigmoid colon and the rectum) interfere primarily in stopping and evacuating salt from food.

Colon cancer is mainly located in the sigmoid region and the colorectal junction (65\% of the localizations) and very rarely in the transverse portion [6].

It supports the cecum being the widest of the human colon allowing the development of the tumors before the symptoms occur. There is obviously a classification of the stages of evolution of these cancers: cancer with a survival period of 5 years (category A) $90 \%$ of the cancers are classified in this type, type D (hepatic metastasis) 5\% types of cancer.

The colonoscope consists of four main parts: the connectors, the universal cord, the clamping system and the distal end [7].

The colonoscope has an operator channel that allows the passage of medical instruments such as for sampling, of coagulation or a laser fiber. Changing the angle of the colonoscope is done with a cable system inserted into the extremities of the device and is driven by two wheels that allow the endoscope head to move in two orthogonal directions that roughly can make all angles of the threeorthogonal system [4].

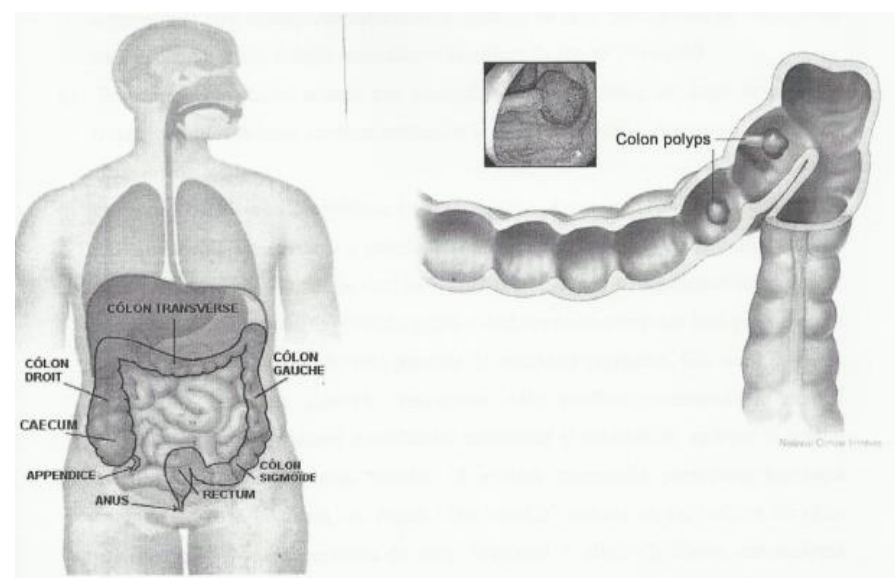

Fig. 1. The Anatomy of the Colon and the Sigmoid Region with Polyp. 


\section{MATHEMATICAL MODEL OF THE COLON}

The paper presents the mathematical realization of the trajectory that the colonoscope should have in the medical intervention, as well as the mathematical demonstration of the functions that make up the colonoscope. We achieved the mathematical model of these functions with Matlab [4].

This simplistic mathematical model of the colon could diminish the perforations achieved by the colonoscope collision with the walls of the colon [2], [3]. Practically these equations could represent a trajectory of the geometric site where the colonoscope would not touch the walls of the intestine, as shown in Fig. 2.

The mathematical model of the colon was performed in four steps:

- We performed the projection on a vertical plane and tried to find the size of the colon.

- We wrote the equations for each portion.

- We split the graph into mathematical continuously functions, and then we set the points of discontinuity.

- We have set the conditions of continuity at the intersection points of the arc.

Mathematical functions:

a) The left curve: The curve is of the form $\mathrm{x}=\mathrm{ay}^{2}-24$, it must intersect points A (-20 -13); B (-20-13).

Final equation is:

$x=\frac{4}{169} y^{2}-24\left\{\begin{array}{l}\frac{13}{2} \sqrt{x+24} \\ -\frac{13}{2} \sqrt{x+24}\end{array}\right.$

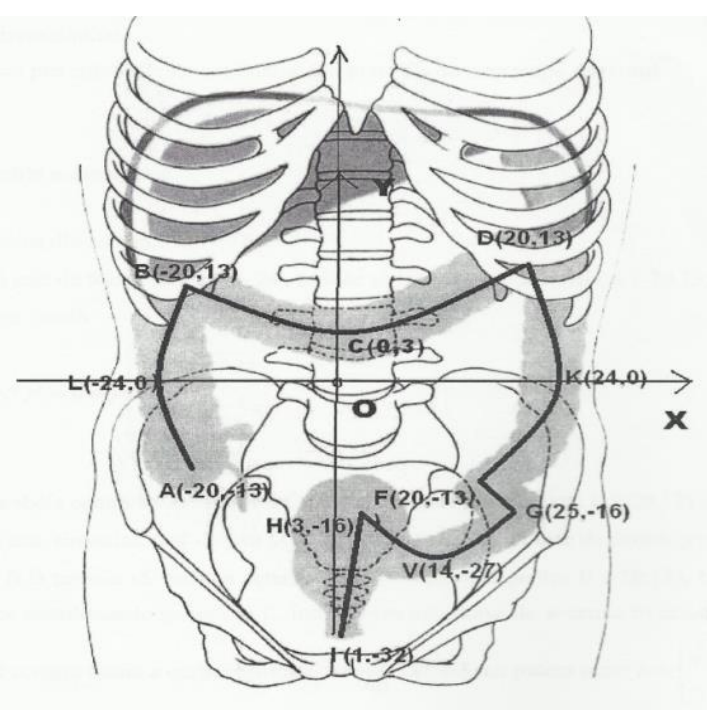

Fig. 2. Colon Mathematical Model.
The parabola is symmetrical with respect to the OY axis, which will result in the shape $\mathrm{y}=\mathrm{ax}^{2}+\mathrm{c}$ but the points $\mathrm{B}, \mathrm{D}$ should verify the equation $=>13=400 a+c$ for $B(-20,13)$, the equation must verify the coordinates of the point $\mathrm{C}$, in the above equation resulting in the final equation of the central curve $y=\frac{1}{40} x^{2}+3$ or we can write:

$x=\left\{\begin{array}{l}\sqrt{40 y-120} \\ -\sqrt{40 y-120}\end{array}\right.$

c) The DFK curve is symmetrical to the ALB parabola resulting in the direct writing of the equation:

$4 \frac{y^{2}}{169}=-x+24$ or $y=\left\{\begin{array}{l}\frac{13}{2} \sqrt{-x+24} \\ -\frac{13}{2} \sqrt{-x+24}\end{array}\right.$

$\mathrm{D}(20$ 13); K(24 0); F(20 -13)

d) FG curve with points F (20 -13) and G (25-16)

$\mathrm{m}_{\mathrm{FG}}=\frac{-16+13}{5}=\frac{3}{5} \Rightarrow \mathrm{y}=-\frac{3}{5} \mathrm{x}-1$

e) The HVG curve is symmetrical with the axis parallel to $\mathrm{OY}$ at the point $\mathrm{V}(14-27)$

The equation is form $\mathrm{y}=\mathrm{ax}^{2}+\mathrm{bx}+\mathrm{c}$ with the tipe $\mathrm{V}$ $\left(-\frac{\mathrm{b}}{2 \mathrm{a}},-\frac{\Delta}{4 \mathrm{a}}\right)$ and $\mathrm{H}(3-16), \mathrm{G}(25-16)$

These two points must verify the initial equation.

The final equation of the HVG curve is:

$y=-\frac{x^{2}}{11}+\frac{28}{11} x-\frac{251}{11}$

f) The IH segment I(1 -32), H(3 -16)

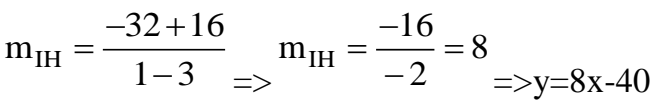

Because the graph was approximated with six mathematical functions, their number should be reduced [5], [8]. According to the mathematical analysis theorem to define a continuous function over an interval, it should be no parallel to the OY axis that intersects the graph into a single point.

- The LBCDK portion can be approximated by a single function by discontinuity points $\mathrm{B}, \mathrm{D}$. The equation of the LB arch is:

$\mathrm{y}=\frac{13}{12} * \sqrt{\mathrm{x}+24}$ (the positive side of BLA arch) 
Equation of DK arch is:

$\mathrm{y}=\frac{13}{12} * \sqrt{\mathrm{x}-24}$ (the positive side of DKF arch)

First of the four continuous functions is:

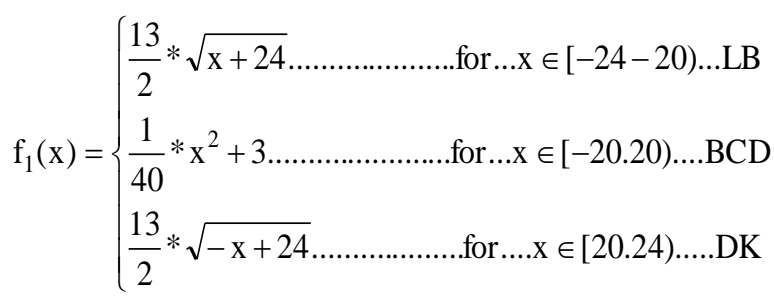

Arch KF of the equation (10) for $\mathrm{x} \in(20,24]$ :

$\mathrm{f} 2(\mathrm{x})=\frac{13}{2} * \sqrt{-\mathrm{x}+24}$

A linear FG for $\mathrm{x} \in[20,24)$ :

$f_{3}(x)=-\frac{3}{5} * x-1$

The fourth continuous functions is:

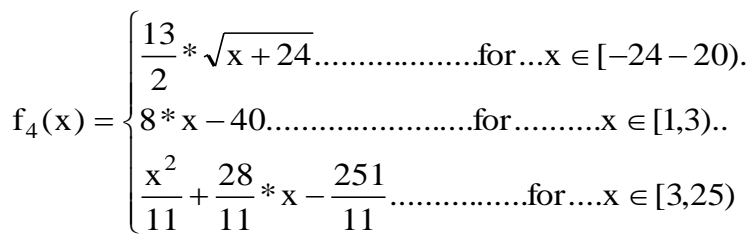

So we approximated the graph with four continuous mathematical functions, as presented in Fig. 3.

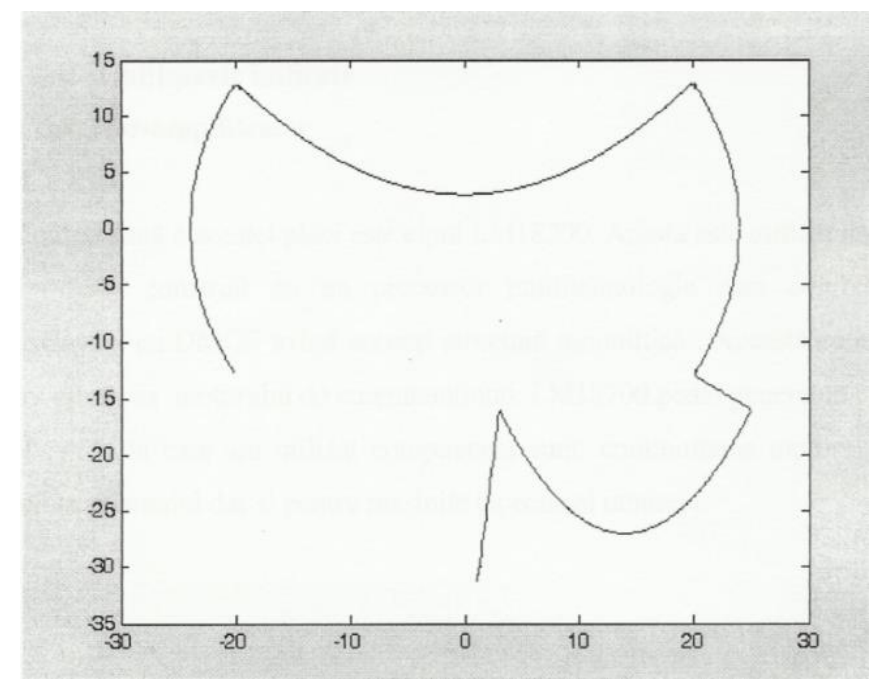

Fig. 3. Graph of the Function Performed in Matlab.

\section{EXPERIMENTAL RESULTS}

The acquisition board of this practical application is called DSPACE DS 1104, this component can deliver in real-time communication between the external environment and the computer. It has a fully programmable processor, and it is possible to program it even with the block diagram programming language (Fig. 4).

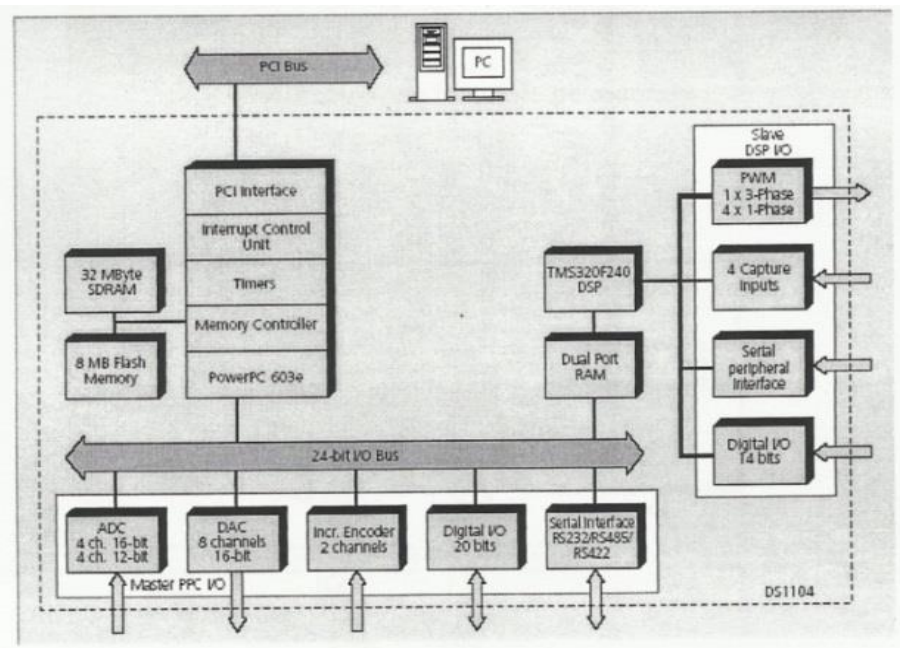

Fig. 4. Block Diagram of DS1104.

At the end of each engine attached to the colonoscope there is an encoder. Incremental encoders are of real use in automated driving processes. They have under construction a light source that penetrates phototransistors in order to have numerical signals on the three channels A, B, I.

The programs of this application are executed in Simulink using Matlab's compiler C and are generated for Control Desk, the latter being a purely experimental program that can handle different parameters and various automation functions.

Control Desk is the central module of this experimental work. Allows the tools listed above to be managed in the most comfortable way possible. This program has a purely experimental goal using Control Desk we can use the same experimental environment for multiple purposes.

Fig. 5 presents the control panel obtained with Control Desk. The acquisition board provides two MLI signals to the converter that feeds the two engines, but at the same time it generates a predefined information of the colonoscope on the predefined trajectory. The two imaginary voltages of circular currents in the two motors are measured by the analogue converter integrated into the acquisition plate. The encoders are connected directly to DSPACE to give the real-time information needed to keep the colonoscope on the predefined trajectory.

In the above diagram from Fig. 6 we can see that it is not necessary for all the blocks to be connected. These were the orders to pilot the colonoscope. In order to be able to make a connection with the Control Desk, we need to give the building command and then reopen with Control Desk. 


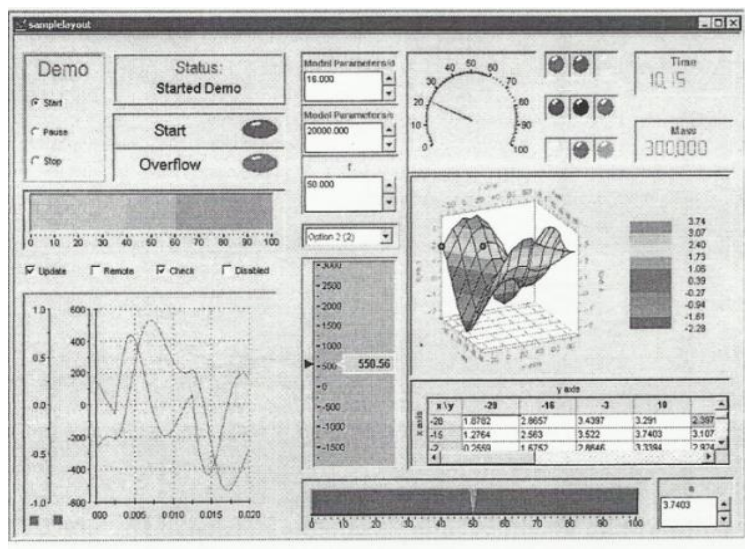

Fig. 5. Control Panel Obtained with Control Desk.

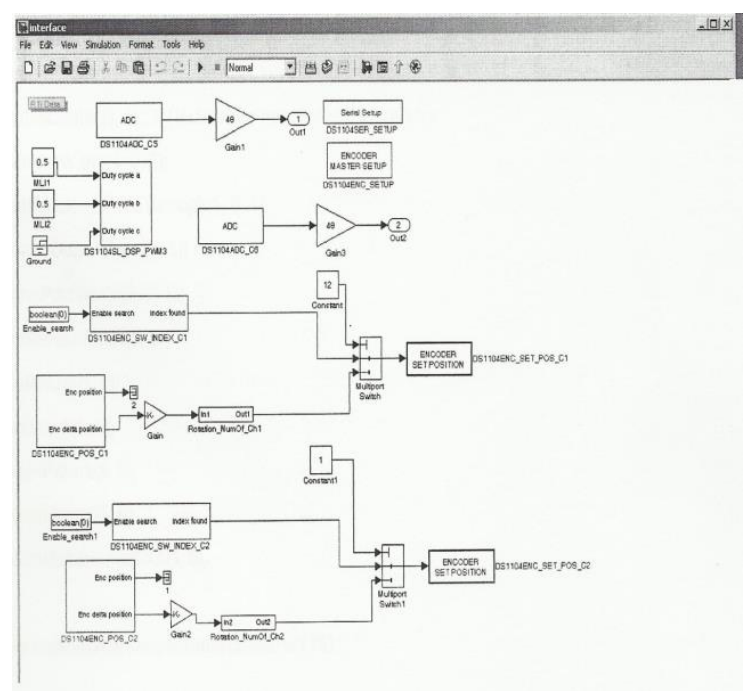

Fig. 6. The Colonoscope Movement with SIMULINK.

After the operating scheme was performed in SIMULINK, a second Control Desk interface was created for changing the direction of colonoscope movement. So, the positioning interface from Fig. 7 was created.

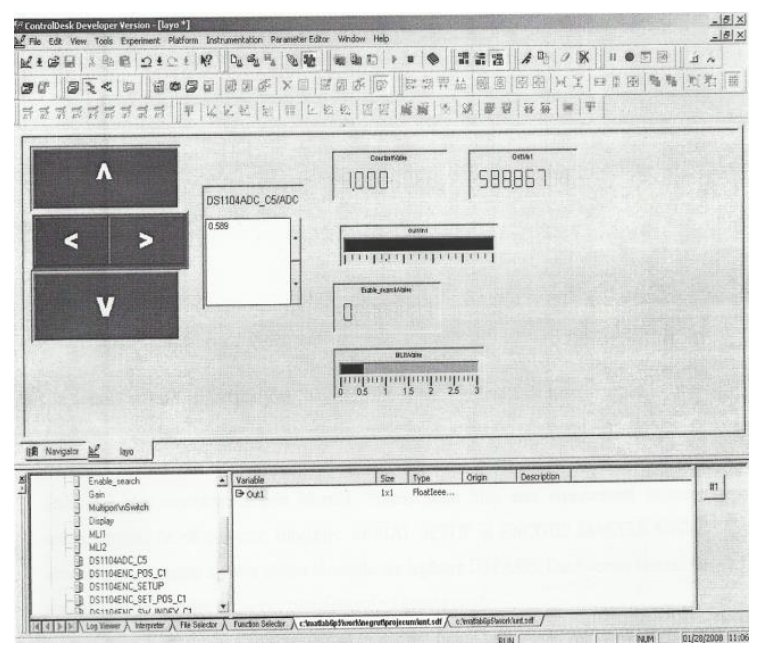

Fig. 7. The Colonoscope Positioning Interface.

\section{CONCLUSIONS}

This paper has achieved the goal of finding a method for reducing the medical doctor's effort, improving the comfort of the patient subjected to a classical colonoscopy and reducing the risk of perforation of the colon.

The central idea of this paper was to achieve the mathematical model of the colon's axes and to try to maintain the colonoscope on the given trajectory. With this hypothesis, we can diminish the contact points with the intestinal walls.

The entire paper was commissioned by an interface created under the Control Desk program that can control the DSpace acquisition board. We have implemented a program that complies with this trajectory, with compiler C in MATLAB.

The practical work was done with 8 Simulink-compatible function blocks and the DSpace library included in this software.

Of course, this mathematical model cannot answer all the problems that exist in the case of a traditional colonoscopy. For better realization of this model, more studies are needed on the human body, to find a common trajectory for all people; there are problems of different conformation of different people.

It is achieved the goal of making an alternative model to traditional colonoscopy and diminishing contact points with the intestinal walls. If there was an emphasis on more scientific research on this subject, there might be a solution to achieve a totally automated or partially computer-controlled colonoscopy. All technical implications in medicine that can improve the condition of the patient and make the medical doctor's task easier can be considered a success.

\section{REFERENCES}

[1] L.M.Matica, C. Gyorodi, H.M. Silaghi, S. Abrudan Caciora, "Real time computation for robotic arm motion upon a linear or circular trajectory", International Journal of Advanced Computer Science and Applications IJACSA, Volume 9, Issue 2, ISSN: 2158-107X, WOS:000426979500004, Pages: 15-19, 2018.

[2] J.Y. Fiset, Human-Machine Interface Design for Process Control Applications, ISA Press, USA, 2008.

[3] L.M. Matica, H. Oros, "Speed computation in movement followed by accurate positioning" International Journal of Computers Communications \& Control, ISSN 1841-9836, 12(1):76-89, 2017

[4] L.M.Matica, C. Gyorodi, H. Silaghi, A. Silaghi, "Mixed profile method of speed and location for robotic arms motion used for precise positioning", International Journal of Advanced Computer Science and Applications IJACSA, Volume 9, Issue 5, 2018.

[5] A. De Sabata, L. Matekovits, A. Silaghi, I. Peter, "Anizotropic dielectric devised by metamaterials-related technique", IEEE 11th International Conference on Communications (COMM), Bucharest, Romania, pp. 133-136, 2016.

[6] O. Fratila, A. Maghiar, T. Ilias, M.A .Silaghi, "Analysis of the colonoscopic findings in patients with the acute gastrointestinal tract bleeding", Journal of Computer Scinence and Control Systems, University of Oradea, Romania, ISSN 1844-6043, pp. 135-140, 2008.

[7] M. Wan, Z. Liang, Q. Ke, L. Hong, "Automatic centerline extraction for virtual colonoscopy", IEEE Transactions on Medical Imaging, Vol.21, Issue 12, Dec.2, ISSN: 0278-0062, pp. 133-136, 2016.

[8] H. Silaghi, V.Spoiala, M.Silaghi, Acţionări electrice, Editura Mediamira, Cluj-Napoca, ISBN 978-973-759-819-6, 314 pp., 2009. 